Research Article

\title{
Infinitely Many Elliptic Solutions to a Simple Equation and Applications
}

\author{
Long Wei and Yang Wang \\ Institute of Applied Mathematics and Engineering Computations, Hangzhou Dianzi University, Zhejiang, Hangzhou 310018, China \\ Correspondence should be addressed to Long Wei; alongwei@gmail.com
}

Received 4 June 2013; Accepted 4 July 2013

Academic Editor: Pavel Kurasov

Copyright (C) 2013 L. Wei and Y. Wang. This is an open access article distributed under the Creative Commons Attribution License, which permits unrestricted use, distribution, and reproduction in any medium, provided the original work is properly cited.

\begin{abstract}
Based on auxiliary equation method and Bäcklund transformations, we present an idea to find infinitely many Weierstrass and Jacobi elliptic function solutions to some nonlinear problems. First, we give some nonlinear iterated formulae of solutions and some elliptic function solutions to a simple auxiliary equation, which results in infinitely many Weierstrass and Jacobi elliptic function solutions of the simple equation. Then applying auxiliary equation method to some nonlinear problems and combining the results with exact solutions of the auxiliary equation, we obtain infinitely many elliptic function solutions to the corresponding nonlinear problems. The employed approach is powerful and can be also applied to solve other nonlinear differential equations.
\end{abstract}

\section{Introduction}

Partial differential equations (PDEs) describe various nonlinear phenomena in natural and applied sciences such as fluid dynamics, plasma physics, solid state physics, optical fibers, acoustics, mechanics, biology, and mathematical finance. Their solution spaces are infinite dimensional and contain diverse solution structures. It is of significant importance to solve nonlinear PDEs from both theoretical and practical points of view. In some decades, many authors are devoted to investigate the exact solutions to nonlinear PDEs. With the help of exact solutions, when they exist, the mechanism of complicated physical phenomena and dynamical processes modeled by these nonlinear PDEs can be better understood. They can also help to analyze the stability of these solutions and to check numerical analysis for these nonlinear PDEs.

In recent years, reducing PDEs into ordinary differential equations (ODEs) has been proved to be a successful idea to generate exact solutions of nonlinear wave equations. Many approaches to exact solutions in the literature follow such an idea, which contain tanh-function method [1-4], sechfunction method $[5,6]$, homogeneous balance method [7, 8], extended tanh-function method [9], Jacobi elliptic function method [10], exp-function method [11], F-expansion method [12], and transformed rational function method [13]. The tanh-function method and the $G^{\prime} / G$ expansion methods are special cases of general Frobenius' idea [14] or the transformed rational function method [13]. Recently, the multiple exp-function was also used to present three waves in $(3+1)$ dimension [15]. There is also a generalized theory of the Bell polynomials [16], which describe generalized bilinear differential equations [17]. Among those, auxiliary equation methods are widely used to find exact solutions (especially elliptic solutions) of PDEs by some authors (see [1,3,18-24], etc.). But in many literatures, the authors constructed exact solutions of PDEs making use of finite solutions of the auxiliary equations employed and have not obtained infinitely many solutions, especially Weierstrass and Jacobi elliptic solutions.

In this work, we will seek for nonlinear iterated formulae of solutions to a simple auxiliary equation. Simultaneously, in order to obtain infinitely many new elliptic solutions of some nonlinear PDEs, we will also give some Weierstrass and Jacobi elliptic function solutions of the simple auxiliary equation. Based on these facts, we can construct infinitely many Weierstrass and Jacobi elliptic function solutions of destination equations. The obtained results are new and introduce useful analysis for equations of identical nonlinearities.

This paper is organized as follows: in Section 2, we provide some nonlinear iterated formulae of solutions and some new elliptic solutions to a simple equation. In Section 3, we give some examples to demonstrate the application of our approach. The remainder of this paper is a discussion. 


\section{Preliminaries}

In this section, we try to find some nonlinear iterated formulae of solutions and infinitely many Weierstrass and Jacobi elliptic function solutions to the auxiliary equation

$$
\left(\frac{d \eta(\xi)}{d \xi}\right)^{2}=a \eta+b \eta^{2}+c \eta^{3}
$$

We will solve some NPDEs by the exact solutions of the auxiliary equation; consequently we can obtain infinitely many Weierstrass and Jacobi elliptic function solutions and periodic and soliton solutions of destination equations.

2.1. Nonlinear Iterated Formulae of Solutions to (1). In order to find infinitely many elliptic solutions to (1), we assume that $a b c \neq 0$.

By the Bäcklund transformations and a modified truncation approach, we can obtain the following nonlinear iterated formulae of solutions; that is, if $w=w(\xi)$ is a solution to (1), then so is $\eta=\eta(\xi)$ :

$$
\begin{aligned}
\eta_{1}= & \frac{a}{c w} \\
\eta_{2}^{ \pm}= & \pm \frac{2 a+\left(b \mp \sqrt{b^{2}-4 a c}\right) w}{\mp b+\sqrt{b^{2}-4 a c} \mp 2 c w} ; \\
\eta_{3}^{ \pm}= & \pm \frac{\mp a b+a \sqrt{b^{2}-4 a c}+\left(\mp b^{2}+b \sqrt{b^{2}-4 a c} \pm 2 a c\right) w}{c\left[2 a+\left(b \mp \sqrt{b^{2}-4 a c}\right) w\right]} \\
\eta_{4}^{ \pm}= & \pm\left(\left[\mp 2 a+\left(\sqrt{b^{2}-4 a c} \mp b\right) w\right]\right. \\
& \times\left(2 c \left(\left[\left(2 a c-b^{2}\right)\left(\sqrt{b^{2}-4 a c} \pm b\right) \pm 2 a b c\right] w\right.\right. \\
\eta_{i}^{ \pm}= & \frac{A_{0, i}+A_{1, i} w+A_{2, i} w^{2}}{B_{0, i}+B_{1, i} w+B_{2, i} w^{2}}, \quad(i=6,7,8,9) \\
\eta_{5}^{ \pm}= & \frac{\left.-a b \pm a \sqrt{b^{2}-4 a c}-4 a c w-c\left(b \pm \sqrt{b^{2}-4 a c}\left(b \pm \sqrt{b^{2}-4 a c}\right)\right)\right)^{-1}}{2 c\left(a+b w+c w^{2}\right)}
\end{aligned}
$$

where

$$
\begin{gathered}
A_{0,6}=\mp R_{6}^{ \pm}, \quad A_{1,6}=1 \mp \frac{b}{a} R_{6}^{ \pm}, \\
A_{2,6}=-\frac{a b\left(b^{2}-3 a c\right) \mp\left(2 a^{2} c^{2}-4 a b^{2} c+b^{4}\right) R_{6}^{ \pm}}{a^{2} c\left(2 a \mp b R_{6}^{ \pm}\right)}, \\
B_{0,6}=1, \quad B_{1,6}=\frac{a b \mp\left(b^{2}-2 a c\right) R_{6}^{ \pm}}{a\left(2 a \pm b R_{6}^{ \pm}\right)},
\end{gathered}
$$$$
B_{2,6}=\frac{a\left(2 a^{2} c^{2}+b^{4}-4 a b^{2} c\right) \mp b\left(5 a^{2} c^{2}-5 a b^{2} c+b^{4}\right) R_{6}^{ \pm}}{a^{2}\left[a\left(b^{2}-2 a c\right) \mp b\left(b^{2}-3 a c\right) R_{6}^{ \pm}\right]}
$$$$
R_{6}^{ \pm}=\frac{ \pm b+\sqrt{b^{2}-4 a c}}{2 c}
$$$$
A_{0,7}= \pm(c+M a) R_{7}^{ \pm}, \quad A_{1,7}=1 \text {, }
$$$$
A_{2,7}=\frac{a M\left[a\left(b^{2}-2 a c\right) \pm b c\left(3 a c-b^{2}\right) R_{7}^{ \pm}\right]}{a b\left(3 a c-b^{2}\right) \pm c\left(2 a^{2} c^{2}-4 a b^{2} c+b^{4}\right) R_{7}^{ \pm}},
$$$$
B_{0,7}=-1-\frac{a}{c} M \text {, }
$$$$
B_{1,7}=\frac{-2 a^{2} M-a c \pm b c(a M+c) R_{7}^{ \pm}}{a c^{2} R_{7}^{ \pm}}, \quad B_{2,7}=M
$$$$
R_{7}^{ \pm}=\frac{ \pm b+\sqrt{b^{2}-4 c a}}{2 c^{2}}, \quad M \text { is an arbitrary constant; }
$$

$$
\begin{gathered}
A_{0,8}=M, \quad A_{1,8}=\mp R_{8}^{ \pm}, \quad A_{2,8}=1 \mp \frac{b}{a} R_{8}^{ \pm}, \\
B_{0,8}=\frac{c\left(2 a \mp b R_{8}^{ \pm}\right) M}{-a b \pm\left(b^{2}-2 a c\right) R_{8}^{ \pm}}, \\
B_{1,8}=1, \quad B_{2,8}=\frac{c\left(2 a \mp b R_{8}^{ \pm}\right)}{a \sqrt{b^{2}-4 a c}},
\end{gathered}
$$

$R_{8}^{ \pm}=\frac{ \pm b+\sqrt{b^{2}-4 a c}}{2 c}, \quad M$ is an arbitrary constant;

$$
A_{0,9}=M, \quad A_{1,9}=\mp N R_{9}^{ \pm} \text {, }
$$$$
A_{2,9}=-\frac{a(b-2 c N)+ \pm c(b N-2 a) R_{9}^{ \pm}}{b^{2}-2 a c-b c N \pm c(2 c N-b) R_{9}^{ \pm}} \text {, }
$$

$$
\begin{gathered}
B_{0,9}=\frac{c\left(2 a \mp b R_{9}^{ \pm}\right) M}{-a b \pm\left(b^{2}-2 a c\right) R_{9}^{ \pm}}, \\
B_{1,9}=N, \quad B_{2,9}=1,
\end{gathered}
$$

$R_{9}^{ \pm}=\frac{ \pm b+\sqrt{b^{2}-4 a c}}{2 c}, \quad M, N$ are two arbitrary constants;

$$
\begin{gathered}
\eta_{10}^{ \pm}= \pm \frac{a\left(\sqrt{b \mp 2 \sqrt{a c}} w+w^{\prime}\right)}{\sqrt{a c}\left(\sqrt{b \mp 2 \sqrt{a c}} w-w^{\prime}\right)} \\
\eta_{11}^{ \pm}=-\frac{A_{1}+A_{2} w \pm A_{3} L w^{\prime}}{c\left(b+2 \sqrt{b^{2}-3 a c}\right)\left(4 a^{2} c-a b^{2} \pm 3 L w^{\prime}\right)}
\end{gathered}
$$

with

$$
\begin{gathered}
A_{1}=18 a^{2} b^{2} c-3 a b^{4}-24 a^{3} c^{2} \\
+3 a b\left(4 a c-b^{2}\right) \sqrt{b^{2}-3 a c} \\
A_{2}=2 a c\left(4 a c-b^{2}\right)\left(b+2 \sqrt{b^{2}-3 a c}\right) \\
A_{3}=6 a c-b^{2}+b \sqrt{b^{2}-3 a c} \\
L=\frac{1}{3} \sqrt{\left(b^{2}-4 a c\right)\left[9 a b c-2 b^{3}+2\left(3 a c-b^{2}\right) \sqrt{b^{2}-3 a c}\right]}
\end{gathered}
$$




$$
\eta_{12}^{ \pm}=-\frac{A_{1}+A_{2} w \pm A_{3} L w^{\prime}}{c\left(-b+2 \sqrt{b^{2}-3 a c}\right)\left(4 a^{2} c-a b^{2} \pm 3 L w^{\prime}\right)},
$$

with

$$
\begin{gathered}
A_{1}=-18 a^{2} b^{2} c+3 a b^{4}+24 a^{3} c^{2} \\
-3 a b\left(4 a c-b^{2}\right) \sqrt{b^{2}-3 a c}, \\
A_{2}=2 a c\left(4 a c-b^{2}\right)\left(-b+2 \sqrt{b^{2}-3 a c}\right), \\
A_{3}=-6 a c+b^{2}+b \sqrt{b^{2}-3 a c}, \\
L=\frac{1}{3} \sqrt{\left(b^{2}-4 a c\right)\left[9 a b c-2 b^{3}-2\left(3 a c-b^{2}\right) \sqrt{b^{2}-3 a c}\right]} ; \\
\eta_{13}^{ \pm}=\frac{A_{1}+A_{2} w \pm A_{3} w^{\prime}}{B_{1} \pm B_{2} w^{\prime}},
\end{gathered}
$$

with

$$
\begin{gathered}
A_{1}=-6 a\left[\left(-b+\sqrt{b^{2}-3 a c}\right)\left(4 b^{4}-27 a b^{2} c+36 a^{2} c^{2}\right)\right. \\
\left.\quad+6 a b c\left(b^{2}-6 a c\right)\right] \\
A_{2}=27 M L^{2}, \quad A_{3}=\frac{9}{c}\left(-b+\sqrt{b^{2}-3 a c}\right) M L \\
B_{1}=8\left(-b+\sqrt{b^{2}-3 a c}\right)\left(27 a^{2} b c^{2}-15 a b^{3} c+2 b^{5}\right) \\
+6 a c\left(4 b^{4}-27 a b^{2} c+36 a^{2} c^{2}\right) \\
B_{2}=27 M L \\
L=\frac{2}{c} \sqrt{b\left(2 b^{2}-9 a c\right)-2\left(b^{2}-3 a c\right)^{3 / 2}} \\
M=c^{2}\left[\left(-b+\sqrt{b^{2}-3 a c}\right)\left(6 a c-2 b^{2}\right)-3 a b c\right] \\
\eta_{14}^{ \pm}=\frac{A_{1}+A_{2} w \pm A_{3} w^{\prime}}{B_{1} \pm B_{2} w^{\prime}}
\end{gathered}
$$

with

$$
\begin{aligned}
A_{1}= & 6 a\left[\left(b+\sqrt{b^{2}-3 a c}\right)\left(4 b^{4}-27 a b^{2} c+36 a^{2} c^{2}\right)\right. \\
& \left.-6 a b c\left(b^{2}-6 a c\right)\right], \\
A_{2}= & 27 M L^{2}, \quad A_{3}=-\frac{9}{c}\left(b+\sqrt{b^{2}-3 a c}\right) M L, \\
B_{1}= & -8\left(b+\sqrt{b^{2}-3 a c}\right)\left(27 a^{2} b c^{2}-15 a b^{3} c+2 b^{5}\right) \\
& +6 a c\left(4 b^{4}-27 a b^{2} c+36 a^{2} c^{2}\right),
\end{aligned}
$$

$$
\begin{gathered}
B_{2}=27 M L, \\
L=\frac{2}{c} \sqrt{b\left(2 b^{2}-9 a c\right)+2\left(b^{2}-3 a c\right)^{3 / 2}}, \\
M=-c^{2}\left[\left(b+\sqrt{b^{2}-3 a c}\right)\left(6 a c-2 b^{2}\right)+3 a b c\right] \\
\eta_{15}=\frac{\sqrt{a c}\left(a+b w+c w^{2}+\sqrt{b-2 \sqrt{a c}} w^{\prime}\right)}{c\left(-a-b w-c w^{2}+\sqrt{b-2 \sqrt{a c}} w^{\prime}\right)} \\
\eta_{16}=\frac{\sqrt{a c}\left(a+b w+c w^{2}-\sqrt{b+2 \sqrt{a c}} w^{\prime}\right)}{c\left(a+b w+c w^{2}+\sqrt{b+2 \sqrt{a c}} w^{\prime}\right)} .
\end{gathered}
$$

Above, we have given finitely many nonlinear iterated formulae of solutions to (1). In fact, it is possible to obtain infinitely many ones by substituting one into another repeatedly. Here, it should be pointed out that the previous iterative procedure can be done finite times for some formulae but infinite times for some other ones. For example, substituting (3) into (2) we can obtain a new nonlinear iterated formula:

$$
\eta_{2-1}^{ \pm}= \pm \frac{a\left[\mp b+\sqrt{b^{2}-4 a c} \mp 2 c w\right]}{c\left[2 a+\left(b \mp \sqrt{b^{2}-4 a c}\right) w\right]},
$$

but repeating the procedure again will result in (3) itself; substituting $\eta_{2}^{-}$into $\eta_{2-1}^{-}$results in $a / \mathrm{cw}$; substituting $\eta_{2}^{+}$into $\eta_{2-1}^{-}$ results in $w$ and so on. But for solutions $\eta_{10}^{ \pm}$and so forth, the iterative procedure are infinite For instance, substituting $\eta_{2}^{ \pm}$ or $\eta_{5}^{ \pm}$into $\eta_{10}^{ \pm}$and iterating the results by $\eta_{10}^{ \pm}$can generate infinitely many iterated formulae of solutions.

2.2. Infinitely Many Weierstrass Elliptic Function Solutions to (1). Let $\wp(\xi)=\wp\left(\xi ; g_{2}, g_{3}\right)$ be the Weierstrass elliptic function satisfying

$$
\wp^{\prime 2}(\xi)=4 \wp^{3}(\xi)-g_{2} \wp(\xi)-g_{3},
$$

where $g_{2}, g_{3}$ are real parameters and called invariants [25], and $\wp^{\prime}(\xi)$ denotes the first-order derivative. It follows from (21) that the second-order derivative is

$$
\wp^{\prime \prime}(\xi)=6 \wp^{2}(\xi)-g_{2} .
$$

From the last previous two equations, we will give some Weierstrass elliptic function solutions to (1), among which there are many new solutions different from those in [26] and some latter references. In fact, in [26] the author considered the following auxiliary equation:

$$
\left(\frac{d \theta}{d \xi}\right)^{2}=d_{0}+d_{2} \theta^{2}+d_{4} \theta^{4}
$$

but this equation can be converted into (1) by the transformation $\eta(\xi)=\theta^{2}(\xi)$, with $a=4 d_{0}, b=4 d_{2}$, and $c=4 d_{4}$. 
From (21) and (22), we have the following Weierstrass elliptic function solutions to (1).

(i) When $g_{2}=\left(b^{2}-3 a c\right) / 12, g_{3}=b\left(9 a c-2 b^{2}\right) / 432$, then

$$
\eta=\frac{3 a}{12 \wp(\xi)-b}, \frac{12 \wp(\xi)-b}{3 c} ;
$$

or

$$
\begin{aligned}
& \eta \\
& =\frac{L_{1} \wp(\xi)-\left(b\left(5 a c-b^{2}\right) L_{1}+a\left(6 a c-b^{2}\right)\right) / 12 c\left(2 a+b L_{1}\right)}{\wp(\xi)-\left(2\left(3 a c-b^{2}\right) L_{1}-a b\right) / 12\left(2 a+b L_{1}\right)}, \\
& \\
& \quad \frac{a\left(\wp(\xi)-\left(2\left(3 a c-b^{2}\right) L_{1}-a b\right) / 12\left(2 a+b L_{1}\right)\right)}{c\left(L_{1} \wp(\xi)-\left(b\left(5 a c-b^{2}\right) L_{1}+a\left(6 a c-b^{2}\right)\right) / 12 c\left(2 a+b L_{1}\right)\right)},
\end{aligned}
$$

where $L_{1}=\left(-b \pm \sqrt{b^{2}-4 a c}\right) / 2 c$.

(ii) When $g_{2}=-11 a c / 64-\left(b^{2}+5 b L_{2}\right) / 48, g_{3}=5 b^{3} /$ $3456-a b c / 512+7\left(b^{2}-3 a c\right) L_{2} / 1152$, then

$$
\eta=\frac{3 a\left(12 \wp(\xi)+b+2 L_{2}\right)}{\left(12 \wp(\xi)+L_{2}\right)^{2}}, \frac{\left(12 \wp(\xi)+L_{2}\right)^{2}}{3 c\left(12 \wp(\xi)+b+2 L_{2}\right)},
$$

where $L_{2}=\left(-5 b \pm 3 \sqrt{b^{2}-4 a c}\right) / 8$.

(iii) When $g_{2}=b^{2} / 192+a c / 16$ and $g_{3}=b\left(36 a c-b^{2}\right) /$ 13824 , then

$$
\begin{aligned}
\eta= & \frac{576 \wp^{\prime 2}(\xi)}{c(24 \wp(\xi)+b)^{2}}, \frac{a(24 \wp(\xi)+b)^{2}}{576 \wp^{\prime 2}(\xi)}, \\
& \frac{b^{2}-36 a c-96 b \wp(\xi)+2304 \wp^{2}(\xi)}{24 c(24 \wp(\xi)+b)}, \\
& \frac{24 a(24 \wp(\xi)+b)}{b^{2}-36 a c-96 b \wp(\xi)+2304 \wp^{2}(\xi)}, \\
& \frac{\left(L_{3} \wp(\xi)-\bar{A}_{1}\right)^{2}}{L_{3}\left(\wp(\xi)+\bar{B}_{1}\right)^{2}}, \frac{a L_{3}\left(\wp(\xi)+\bar{B}_{1}\right)^{2}}{c\left(L_{3} \wp(\xi)-\bar{A}_{1}\right)^{2}}
\end{aligned}
$$

with $\bar{A}_{1}=\left(4 a b+\left(12 a c-b^{2}\right) L_{3}\right) / 48\left(b+2 c L_{3}\right), \bar{B}_{1}=(4 a b-$ $\left.\left(12 a c-5 b^{2}\right) L_{3}\right) / 48\left(2 a+b L_{3}\right)$, and $L_{3}=\left(-b \pm \sqrt{b^{2}-4 a c}\right) / 2 c$;

$$
\eta=\frac{L_{3}\left(\wp(\xi)-\bar{A}_{2}\right)^{2}}{\left(\wp(\xi)-\bar{B}_{2}\right)^{2}}, \frac{a\left(\wp(\xi)-\bar{B}_{2}\right)^{2}}{c L_{3}\left(\wp(\xi)-\bar{A}_{2}\right)^{2}}
$$

with $\bar{A}_{2}=\left(a\left(12 a c-b^{2}\right)+b\left(8 a c-b^{2}\right) L_{3}\right) / 48\left[\left(2 a c-b^{2}\right) L_{3}-a b\right]$, $\bar{B}_{2}=\left(a b\left(5 b^{2}-16 a c\right)+\left(5 b^{4}+12 a^{2} c^{2}-21 a b^{2} c\right) L_{3}\right) / 48[a(2 a c-$ $\left.\left.b^{2}\right)+b\left(3 a c-b^{2}\right) L_{3}\right]$, and $L_{3}=\left(-b \pm \sqrt{b^{2}-4 a c}\right) / 2 c$; or

$$
\begin{aligned}
\eta= & \frac{\left(\bar{A}_{3} \wp(\xi)+\bar{B}_{3}+E \wp^{\prime}(\xi)\right)^{2}}{\left(\bar{A}_{4} \wp(\xi)+\bar{B}_{4}-D \wp^{\prime}(\xi)\right)^{2}}, \\
& \frac{a\left(\bar{A}_{4} \wp(\xi)+\bar{B}_{4}-D \wp^{\prime}(\xi)\right)^{2}}{c\left(\bar{A}_{3} \wp(\xi)+\bar{B}_{3}+E \wp^{\prime}(\xi)\right)^{2}}
\end{aligned}
$$

with $\bar{A}_{3}=D\left(2 a D^{2}+b E^{2}\right) \bar{L}, \bar{B}_{3}=(D / 48)\left(12 a c E^{2}-b^{2} E^{2}+\right.$ $\left.4 a b D^{2}\right) \bar{L}, \bar{A}_{4}=E\left(b D^{2}+2 c E^{2}\right) \bar{L}$, and $\bar{B}_{4}=(E / 48)\left(12 a c D^{2}-\right.$ $\left.b^{2} D^{2}+4 b c E^{2}\right) \bar{L}$, where $\bar{L}= \pm\left(1 / 2 \sqrt{c E^{4}+b E^{2} D^{2}+a D^{4}}\right)$, and $D, E$ are two arbitrary constants.

Note that in any pair of solutions, the latter can be obtained from the former through formula (2). Indeed, combining solutions (24)-(31) with the nonlinear iterated formulae (2)-(20) and their infinitely many derivatives can yield infinitely many Weierstrass elliptic function solutions of (1).

2.3. Infinitely Many Jacobi Elliptic Function Solutions to (1). Assume that $K, C$, and $D$ are arbitrary constants, and $m \in$ $(0,1)$ is the elliptic modulus; then (1) has the following Jacobi elliptic solutions.

(I) $a=4 K, b=-4\left(1+m^{2}\right), c=4 m^{2} / K$,

$$
\begin{aligned}
\eta= & K \operatorname{cd}(\xi, m)^{2}, K \operatorname{sn}(\xi, m)^{2}, \\
& \frac{K}{m^{2}} \mathrm{dc}(\xi, m)^{2}, \frac{K}{m^{2}} \mathrm{~ns}(\xi, m)^{2} .
\end{aligned}
$$

Indeed, the former two solutions and the latter two ones can transform each other by formula (2). In what follows, we omit the solutions that can be generated by formula (2).

(II) $a=4 K\left(1-m^{2}\right), b=-4\left(1-2 m^{2}\right), c=-4 m^{2} / K$,

$$
\eta=K \operatorname{cn}(\xi, m)^{2}, K\left(1-m^{2}\right) \operatorname{sd}(\xi, m)^{2} .
$$

(III) $a=-4 K\left(1-m^{2}\right), b=4\left(2-m^{2}\right), c=-4 / K$,

$$
\eta=K \operatorname{dn}(\xi, m)^{2},-K \operatorname{cs}(\xi, m)^{2} \text {. }
$$

(IV) $a=K\left(m^{2}-1\right), b=2\left(1+m^{2}\right), c=\left(m^{2}-1\right) / K$,

$$
\begin{aligned}
\eta^{ \pm}= & K[\operatorname{nd}(\xi, m) \pm m \operatorname{sd}(\xi, m)]^{2}, \\
& -K[\operatorname{nc}(\xi, m) \pm(\xi, m)]^{2}, \\
& \frac{K}{1-m^{2}}[\operatorname{dn}(\xi, m) \pm m \mathrm{cn}(\xi, m)]^{2}, \\
& \frac{K}{m^{2}-1}[\operatorname{cs}(\xi, m) \pm \operatorname{ds}(\xi, m)]^{2} .
\end{aligned}
$$

$(\mathrm{V}) a=\left(\left(C^{2}-D^{2}\right) / K\right)\left(m^{2}-1\right), b=2\left(1+m^{2}\right), c=$ $-\left(K /\left(C^{2}-D^{2}\right)\right)\left(m^{2}-1\right)$,

$$
\eta^{ \pm}=\frac{[D \operatorname{cn}(\xi, m)+C \operatorname{dn}(\xi, m)]^{2}}{K\left[1 \pm \sqrt{1+C^{2}\left(1-m^{2}\right) /\left(D^{2}-C^{2}\right)} \operatorname{sn}(\xi, m)\right]^{2}}
$$

(VI) $a=K, b=2\left(-2+m^{2}\right), c=m^{4} / K$,

$$
\begin{aligned}
\eta^{ \pm}= & \frac{K \operatorname{cn}(\xi, m)^{2}}{\left[\sqrt{1-m^{2}} \pm \operatorname{dn}(\xi, m)\right]^{2}}, \\
& \frac{K \operatorname{sn}(\xi, m)^{2}}{[1 \pm \operatorname{dn}(\xi, m)]^{2}}, \\
& \frac{K}{m^{2}}\left[\operatorname{cd}(\xi, m) \pm i \sqrt{1-m^{2}} \operatorname{sd}(\xi, m)\right]^{2}, \\
& -\frac{K}{m^{2}}[\operatorname{cn}(\xi, m) \pm i \sin (\xi, m)]^{2} .
\end{aligned}
$$


(VII) $a=K m^{2} /\left(C^{2}-D^{2}\right), b=2\left(-2+m^{2}\right), c=\left(C^{2}-\right.$ $\left.D^{2}\right) m^{2} / K$,

$$
\begin{aligned}
\eta^{ \pm}= & -\left(K[C+D \operatorname{dn}(\xi, m)]^{2}\right) \\
& \times\left(\left(C^{2}-D^{2}\right)^{2}\right. \\
& \times\left[\operatorname{cn}(\xi, m) \pm \sqrt{\left.\left.\frac{D^{2} m^{2}}{D^{2}-C^{2}}-1 \operatorname{sn}(\xi, m)\right]^{2}\right)^{-1}}\right.
\end{aligned}
$$

(VIII) $a=K, b=2\left(1-2 m^{2}\right), c=1 / K$,

$$
\begin{aligned}
\eta^{ \pm}= & K[\mathrm{~ns}(\xi, m) \pm \mathrm{cs}(\xi, m)]^{2} \\
& K[m \operatorname{sn}(\xi, m) \pm i \operatorname{dn}(\xi, m)]^{2} \\
& K\left[\operatorname{dc}(\xi, m) \pm \sqrt{1-m^{2}}(\xi, m)\right]^{2} \\
& K\left[\operatorname{mcd}(\xi, m) \pm i \sqrt{1-m^{2}} \mathrm{nd}(\xi, m)\right]^{2}
\end{aligned}
$$

(IX) $a=K m^{2} /\left(C^{2}-D^{2}\right), b=2\left(1-2 m^{2}\right), c=\left(C^{2}-\right.$ $\left.D^{2}\right) m^{2} / K$,

$$
\eta^{ \pm}=-\frac{K\left[\operatorname{dn}(\xi, m) \pm \sqrt{D^{2} /\left(D^{2}-C^{2}\right)-m^{2}} \operatorname{sn}(\xi, m)\right]^{2}}{[C+\operatorname{Dcn}(\xi, m)]^{2}} .
$$

(X) $a=K(1-m)^{2}, b=2\left(1+6 m+m^{2}\right), c=(1-m)^{2} / K$

$$
\eta^{ \pm}=-\frac{K[1 \pm \sqrt{m} \mathrm{sn}(\xi, m)]^{2}}{[1 \mp \sqrt{m} \mathrm{sn}(\xi, m)]^{2}}
$$

(XI) $a^{ \pm}= \pm 16 K m, b^{ \pm}=-4\left(1 \pm 6 m+m^{2}\right), c^{ \pm}=(1 \pm m)^{2} / K$

$$
\eta^{ \pm}=\frac{K\left[1 \mp m \mathrm{sn}(\xi, m)^{2}\right]^{2}}{\mathrm{cn}(\xi, m)^{2} \operatorname{dn}(\xi, m)^{2}}, \frac{K[\mathrm{~ns}(\xi, m) \pm m \mathrm{sn}(\xi, m)]^{2}}{(1-m)^{2}}
$$

(XII) $a^{ \pm}= \pm 16 K \sqrt{1-m^{2}}, b^{ \pm}=4\left(2-m^{2} \pm 6 \sqrt{1-m^{2}}\right)$, $c^{ \pm}=4\left(2-m^{2} \pm 2 \sqrt{1-m^{2}}\right) / K$,

$$
\begin{aligned}
\eta^{ \pm}= & -\frac{K\left[\operatorname{cn}(\xi, m)^{2} \pm \sqrt{1-m^{2}} \operatorname{sn}(\xi, m)^{2}\right]^{2}}{\operatorname{dn}(\xi, m)^{2}}, \\
& -\frac{K\left[\operatorname{dn}(\xi, m) \pm \sqrt{1-m^{2}} \mathrm{nd}(\xi, m)\right]^{2}}{2-m^{2} \pm 2 \sqrt{1-m^{2}}}
\end{aligned}
$$

$$
\begin{aligned}
& \frac{K}{m^{4} \operatorname{sn}(\xi, m)^{2}} \\
& \times\left[\left(1-m^{2} \mp \sqrt{1-m^{2}}\right) \mathrm{nc}(\xi, m)+m^{2} \mathrm{cn}(\xi, m)\right]^{2}, \\
& \frac{K}{m^{4} \operatorname{sn}(\xi, m)^{2} \operatorname{cn}(\xi, m)^{2}}\left[\operatorname{dn}(\xi, m)^{2} \mp \sqrt{1-m^{2}}\right]^{2} .
\end{aligned}
$$

Combining solutions (32)-(43) with the nonlinear iterated formulae (2)-(20) and their derivatives can give infinitely many Jacobi elliptic function solutions of (1), and these solutions can be degenerated to solutions with hyperbolic or trigonometric functions as long as letting $m$ go to 1 or 0 .

\section{Infinitely Many Elliptic Solutions of Some Nonlinear PDEs}

Now we apply auxiliary equation methods to some nonlinear PDEs and obtain their infinitely many elliptic function solutions.

Example 1. First we consider the Kadomtsev-Petviashvili (KP) equation

$$
\left(u_{t}-6 u u_{x}+u_{x x x}\right)_{x}+3 u_{y y}=0
$$

which was introduced in 1970 [27] and is of both physical and mathematical interests. By using the wave variable $\xi=\alpha x+$ $\beta y+\gamma t$ and integrating twice, the KP equation (44) can be converted to the ODE

$$
\alpha^{4} u^{\prime \prime}+\left(\alpha \gamma+3 \beta^{2}\right) u-3 \alpha^{2} u^{2}=0
$$

if we let the integration constants be zeros.

Assume that the solutions of (45) have the following form:

$$
u(\xi)=\frac{a_{0}+a_{1} \eta(\xi)+a_{2} \eta^{2}(\xi)}{b_{0}+b_{1} \eta(\xi)},
$$

where $\eta(\xi)$ satisfies $(1), a_{i}(i=0,1,2)$ and $b_{j}(j=0,1)$ are constants to be determinated. Substituting (46) into (45) and equating corresponding coefficients of $\eta(\xi)$ to zero yield an algebraic system of $a_{i}, b_{j}, \alpha, \beta$, and $\gamma$. Solving this system with the aid of Maple, we obtain the following solutions of (45):

$$
\begin{aligned}
& u_{\mathrm{I}}(\xi)=\alpha^{2}\left(\frac{b \pm \sqrt{b^{2}-3 a c}}{6}+\frac{c \eta(\xi)}{2}\right), \\
& u_{\mathrm{II}}(\xi)=\alpha^{2}\left(\frac{b \pm \sqrt{b^{2}-3 a c}}{6}+\frac{a}{2 \eta(\xi)}\right), \\
& u_{\mathrm{III}}(\xi)=\frac{c \alpha^{2}\left[a+2 b \eta(\xi)+3 c \eta^{2}(\xi)\right]}{2\left[b \mp \sqrt{b^{2}-3 a c}+3 c \eta(\xi)\right]},
\end{aligned}
$$

where $\eta(\xi)$ satisfies (1), with $\gamma=\left(-3 \beta^{2} \pm \alpha^{4} \sqrt{b^{2}-3 a c}\right) / \alpha$, and $\alpha, \beta, a, b$, and $c$ are arbitrary constants.

$$
u_{\mathrm{IV}}(\xi)=\alpha^{2}\left(\frac{b \pm \sqrt{b^{2}+12 a c}}{6}+\frac{a}{2 \eta(\xi)}+\frac{c \eta(\xi)}{2}\right),
$$


where $\eta(\xi)$ satisfies (1), with $\gamma=\left(-3 \beta^{2} \pm \alpha^{4} \sqrt{b^{2}+12 a c}\right) / \alpha, \alpha$, $\beta, a, b, c$ are arbitrary constants.

Now substituting the solutions $\eta$ 's of (1) into (47) and (48), we obtain immediately infinitely many Weierstrass and Jacobi elliptic solutions of (44), and these elliptic solutions can be degenerated to solutions with hyperbolic or trigonometric functions as long as letting $m$ go to 1 or 0 .

For instance, from (48), (6), (9), and (24) (or (35)) we can obtain infinitely many Weierstrass or Jacobi elliptic solutions of (44) as follows:

$$
\begin{gathered}
u_{n}(x, y, t)=\alpha^{2}\left(\frac{b \pm \sqrt{b^{2}+12 a c}}{6}+\frac{a}{2 \eta_{n}}+\frac{c \eta_{n}}{2}\right), \\
(n=1,2, \ldots), \\
\eta_{n+1}^{ \pm}= \pm \frac{a\left(\sqrt{b \mp 2 \sqrt{a c}} \eta_{n}+\eta_{n}^{\prime}\right)}{\sqrt{a c}\left(\sqrt{b \mp 2 \sqrt{a c}} \eta_{n}-\eta_{n}^{\prime}\right)} \\
\eta_{1}^{ \pm}= \pm \frac{a\left(\sqrt{b \mp 2 \sqrt{a c}} w_{1}+w_{1}^{\prime}\right)}{\sqrt{a c}\left(\sqrt{b \mp 2 \sqrt{a c}} w_{1}-w_{1}^{\prime}\right)} \\
w_{1}=\frac{-a b \pm a \sqrt{b^{2}-4 a c}-4 a c w_{0}-c\left(b \pm \sqrt{b^{2}-4 a c}\right) w_{0}^{2}}{2 c\left(a+b w_{0}+c w_{0}^{2}\right)} .
\end{gathered}
$$

(i) For Weierstrass elliptic function solutions,

$$
w_{0}=\frac{3 a}{12 \wp\left(\xi ; g_{2}, g_{3}\right)-b},
$$

with

$$
g_{2}=\frac{b^{2}-3 a c}{12}, \quad g_{3}=\frac{b\left(9 a c-2 b^{2}\right)}{432},
$$

where $a, b$, and $c$ are arbitrary constants.

(ii) For Jacobi elliptic function solutions,

$$
w_{0}=-K[\mathrm{nc}(\xi, m) \pm \mathrm{sc}(\xi, m)]^{2}
$$

with

$$
\begin{gathered}
a=K\left(m^{2}-1\right), \quad b=2\left(1+m^{2}\right), \quad c=\frac{\left(m^{2}-1\right)}{K}, \\
\gamma=\frac{-3 \beta^{2} \pm \alpha^{4} \sqrt{\left(2+2 m^{2}\right)^{2}+12\left(m^{2}-1\right)^{2}}}{\alpha}, \\
\xi=\alpha x+\beta y+\gamma t,
\end{gathered}
$$

where $\alpha, \beta$, and $K$ are arbitrary constants and $m \in$ $(0,1)$ is the elliptic modulus. If $m$ tends to 1 or 0 , we can also obtain multiple soliton or periodic solutions.
Example 2. Next we investigate infinitely many elliptic function solutions to a negative order KdV equation

$$
\left(\frac{u_{x x}}{u}\right)_{t}+2 u u_{x}=0
$$

which actually comes from the negative KdV hierarchy and can be transformed to the Camassa-Holm equation through a gauge transform (see [28]). The negative order KdV equation (54) will be transformed to the ODE

$$
\alpha \gamma u^{\prime \prime}+u^{3}+\lambda u=0
$$

upon using wave variable $\xi=\alpha x+\gamma t$ and integrating once, where $\lambda$ is a integration constant to be determinated. Similar to the procedure of Example 1, one can get solutions of (55) as follows,

$$
\begin{array}{r}
u(\xi)= \pm \frac{\sqrt{\alpha \gamma\left[-b+2(-1)^{j-1} \sqrt{a c}\right]}\left[\sqrt{a c}-(-1)^{j-1} c \eta(\xi)\right]}{\sqrt{2}\left[\sqrt{a c}+(-1)^{j-1} c \eta(\xi)\right]}, \\
(j=1,2)
\end{array}
$$

where $\eta(\xi)$ satisfies (1), with $\lambda=\alpha \gamma b / 2, \alpha, \gamma, a, b, c$ are arbitrary constants.

Similarly, substituting the solutions $\eta$ 's of (1) into (56), we obtain infinitely many Weierstrass and Jacobi elliptic solutions of (54), among which including hyperbolic and trigonometric functions solutions as long as letting $m$ go to 1 or 0 .

For instance, from (56), (3), (9) and (27) (or (34)) we can obtain infinitely many Weierstrass or Jacobi elliptic solutions of (54) as follows

$$
\begin{gathered}
u_{n}(x, t)= \pm \frac{\sqrt{\alpha \gamma\left[-b+2(-1)^{j-1} \sqrt{a c}\right]}\left[\sqrt{a c}-(-1)^{j-1} c \eta_{n}\right]}{\sqrt{2}\left[\sqrt{a c}+(-1)^{j-1} c \eta_{n}\right]}, \\
\eta_{n+1}= \pm \frac{a\left(\sqrt{b \mp 2 \sqrt{a c}} \eta_{n}+\eta_{n}^{\prime}\right)}{\sqrt{a c}\left(\sqrt{b \mp 2 \sqrt{a c}} \eta_{n}-\eta_{n}^{\prime}\right)}, \\
\eta_{1}^{ \pm}= \pm \frac{(n=0,1,2, \ldots, j=1,2),}{\sqrt{a c}\left(\sqrt{b \mp 2 \sqrt{a c}} w_{1}-w_{1}^{\prime}\right)} \\
w_{1}^{ \pm}= \pm \frac{2 a+\left(b \mp \sqrt{b^{2}-4 a c}\right) w_{0}}{\mp b+\sqrt{b^{2}-4 a c} \mp 2 c w_{0}},
\end{gathered}
$$

(i) for Weierstrass elliptic function solutions,

$$
w_{0}=\frac{576 \wp^{\prime 2}\left(\xi ; g_{2}, g_{3}\right)}{c\left(24 \wp\left(\xi ; g_{2}, g_{3}\right)+b\right)^{2}}
$$


with

$$
g_{2}=\frac{b^{2}}{192}+\frac{a c}{16}, \quad g_{3}=\frac{b\left(36 a c-b^{2}\right)}{13824},
$$

where $a, b$ and $c$ are arbitrary constants;

(ii) for Jacobi elliptic function solutions,

$$
w_{0}=-K \operatorname{cs}(\xi, m)^{2},
$$

with

$$
\begin{gathered}
a=4 K\left(-1+m^{2}\right), \quad b=4\left(2-m^{2}\right), \quad c=-\frac{4}{K}, \\
\lambda=\frac{\alpha \gamma\left(8-4 m^{2}\right)}{2},
\end{gathered}
$$

where $\alpha, \gamma, K$ are arbitrary constants and $m \in(0,1)$ is the elliptic modulus. If $m$ tends to 1 or 0 , we can also obtain multiple soliton or periodic solutions.

Example 3. We consider the two-dimensional Davey-Stewartson equation

$$
\begin{gathered}
i u_{t}+u_{x x}-u_{y y}-2|u|^{2} u-2 u v=0 \\
v_{x x}+v_{y y}+2\left(|u|^{2}\right)_{x x}=0 .
\end{gathered}
$$

This equation is completely integrable and often used to describe the long time evolution of a two-dimensional wave packet $[29,30]$. In recent years, various methods such as inverse scattering, Darboux transformation, variable separation, and Bäcklund transformation have been used to solve the equation, respectively. In this work, we try to deal with (62) by new idea and give it infinitely many Weierstrass and Jacobi elliptic-like solutions.

For our purpose, we introduce the following transformations:

$$
\begin{gathered}
u(x, t)=U(\xi) e^{i \theta}, \\
v(x, t)=V(\xi), \\
\theta=p x+q y+r t, \\
\xi=k x+d y-2(p k-d q) t .
\end{gathered}
$$

Then system (62) is reduced to the following system of ordinary differential equations:

$$
\begin{gathered}
\left(q^{2}-p^{2}-r\right) U+\left(k^{2}-d^{2}\right) U^{\prime \prime}-2 U^{3}-2 U V=0, \\
\left(k^{2}+d^{2}\right) V^{\prime \prime}+2 k^{2}\left(U^{2}\right)^{\prime \prime}=0 .
\end{gathered}
$$

Integrating the second equation in (64) and neglecting the constants of integration we find that

$$
V=-\frac{2 k^{2}}{k^{2}+d^{2}} U^{2}
$$

Substituting (65) into the first equation of the system leads to

$$
U^{\prime \prime}+\frac{q^{2}-p^{2}-r}{k^{2}-d^{2}} U+\frac{2}{k^{2}+d^{2}} U^{3}=0
$$

where prime denotes differentiation with respect to $\xi$. Further multiplying (66) by $U^{\prime}(\xi)$ and integrating it, we obtain that

$$
U^{\prime 2}+\frac{q^{2}-p^{2}-r}{k^{2}-d^{2}} U^{2}+\frac{1}{k^{2}+d^{2}} U^{4}-H=0,
$$

which is regrouped as follows:

$$
U^{\prime 2}=-\frac{1}{k^{2}+d^{2}} U^{4}+\frac{p^{2}+r-q^{2}}{k^{2}-d^{2}} U^{2}+H,
$$

where $H$ is an arbitrary constant of integration.

In order to write (68) in a simple form that we want, let us introduce the following transformation:

$$
\eta(\xi)=U^{2}(\xi) \text {. }
$$

Then (68) can be written as (1) with

$$
a=4 H, \quad b=\frac{4\left(p^{2}+r-q^{2}\right)}{k^{2}-d^{2}}, \quad c=-\frac{4}{k^{2}+d^{2}} .
$$

Now from the solutions $\eta$ 's of (1) and taking into account (63), (65), and (69), we obtain infinitely many Weierstrass and Jacobi elliptic-like solutions to the two-dimensional DaveyStewartson equation (62) as follows:

$$
\begin{gathered}
u_{n}(x, t)=U_{n}(\xi) e^{i \theta}, \quad v_{n}(x, t)=-\frac{2 k^{2}}{k^{2}+d^{2}} U_{n}^{2}(\xi), \\
\quad(n=1,2, \ldots), \\
U_{n}^{2}(\xi)=\eta_{n}(\xi),
\end{gathered}
$$

where $\theta=p x+q y+r t, \xi=k x+d y-2(p k-d q) t$; taking account of (70), $p, q, r, k, d$, and $H$ satisfy the relations such that (1) has an elliptic solution.

For instance, from (6), (9), and (24) (or (35)) we can obtain infinitely many Weierstrass or Jacobi elliptic-like solutions to the two-dimensional Davey-Stewartson equation (62) as follows:

$$
\begin{array}{r}
u_{n}(x, t)=U_{n}(\xi) e^{i \theta}, \quad v_{n}(x, t)=-\frac{2 k^{2}}{k^{2}+d^{2}} U_{n}^{2}(\xi), \\
(n=0,1,2, \ldots),
\end{array}
$$

$$
\begin{gathered}
U_{n}^{2}(\xi)=\eta_{n}(\xi) \\
\eta_{n+1}=-\frac{a\left(\sqrt{b+2 \sqrt{a c} \eta_{n}}+\eta_{n}^{\prime}\right)}{\sqrt{a c}\left(\sqrt{b+2 \sqrt{a c}} \eta_{n}-\eta_{n}^{\prime}\right)} \\
\eta_{0}=\left(-a b \pm a \sqrt{b^{2}-4 a c}-4 a c w_{0}\right. \\
\left.-c\left(b \pm \sqrt{b^{2}-4 a c}\right) w_{0}^{2}\right) \\
\times\left(2 c\left(a+b w_{0}+c w_{0}^{2}\right)\right)^{-1}
\end{gathered}
$$


(i) For Weierstrass elliptic function solutions,

$$
w_{0}=-\frac{k^{2}+d^{2}}{3}\left(6 \wp\left(\xi ; g_{2}, g_{3}\right)-\frac{p^{2}+r-q^{2}}{k^{2}-d^{2}}\right) \text {, }
$$

with

$$
\begin{gathered}
\theta=p x+q y+r t, \\
\xi=k x+d y-2(p k-d q) t, \\
g_{2}=\frac{4 H}{k^{2}+d^{2}}+\frac{4\left(p^{2}+r-q^{2}\right)^{2}}{3\left(k^{2}-d^{2}\right)^{2}}, \\
g_{3}=\frac{4\left(q^{2}-p^{2}-r\right)}{27\left(k^{2}-d^{2}\right)}\left(\frac{\left(p^{2}+r-q^{2}\right)^{2}}{\left(k^{2}-d^{2}\right)^{2}}+\frac{9 H}{k^{2}+d^{2}}\right), \\
a=4 H, \quad b=\frac{4\left(p^{2}+r-q^{2}\right)}{k^{2}-d^{2}}, \quad c=-\frac{4}{k^{2}+d^{2}},
\end{gathered}
$$

where $p, q, r, k, d$, and $H$ are arbitrary real constants.

(ii) For Jacobi elliptic function solutions,

$$
w_{0}=\frac{1}{4}\left(k^{2}+d^{2}\right)\left(1-m^{2}\right)(\operatorname{nd}(\xi, m) \pm m \mathrm{sd}(\xi, m))^{2}
$$

with

$$
\begin{gathered}
\theta=p x+q y+r t, \\
\xi=k x+d y-2(p k-d q) t, \\
a=-\frac{1}{4}\left(1-m^{2}\right)^{2}\left(k^{2}+d^{2}\right), \\
b=2\left(1+m^{2}\right), \quad c=-\frac{4}{k^{2}+d^{2}}, \\
r=\frac{1}{2}\left(1+m^{2}\right)\left(k^{2}-d^{2}\right)+q^{2}-p^{2},
\end{gathered}
$$

where $d, k, p$, and $q$ are arbitrary constants and $m \in$ $(0,1)$ is the elliptic modulus. If $m$ tends to 1 or 0 , we can also obtain multiple soliton or periodic solutions.

\section{Discussion}

In this paper, we have obtained some nonlinear iterated formulae of solutions to the simple equation (1) and its infinitely many Weierstrass and Jacobi elliptic function solutions. Based on this fact, we can obtain infinitely many Weierstrass and Jacobi elliptic function solutions of some nonlinear equations. Our results suggest that the proposed method is reliable and effective to find infinitely many elliptic solutions to some nonlinear problems. The method is concise, and it can also be applied to other kinds of nonlinear equations in mathematical physics.

\section{Acknowledgments}

The first author is partly supported by Zhejiang Provincial Natural Science Foundation of China under Grant no. LY12A01003 and Subjects Research and Development Foundation of Hangzhou Dianzi University under Grant no. ZX100204004-6. The second author is partly supported by NSFC under Grant no. 11101111.

\section{References}

[1] E. J. Parkes and B. R. Duffy, "An automated tanh-function method for finding solitary wave solutions to non-linear evolution equations," Computer Physics Communications, vol. 98, no. 3, pp. 288-300, 1996.

[2] W. Malfliet and W. Hereman, "The tanh method. I. Exact solutions of nonlinear evolution and wave equations," Physica Scripta, vol. 54, no. 6, pp. 563-568, 1996.

[3] E. Fan, "Extended tanh-function method and its applications to nonlinear equations," Physics Letters A, vol. 277, no. 4-5, pp. 212$218,2000$.

[4] A.-M. Wazwaz, "The tanh method for traveling wave solutions of nonlinear equations," Applied Mathematics and Computation, vol. 154, no. 3, pp. 713-723, 2004.

[5] W. X. Ma, "Travelling wave solutions to a seventh order generalized KdV equation," Physics Letters A, vol. 180, no. 3, pp. 221224, 1993.

[6] E. J. Parkes, Z. Zhu, B. R. Duffy, and H. C. Huang, "Sechpolynomial travelling solitary-wave solutions of odd-order generalized KdV equations," Physics Letters A, vol. 248, no. 2-4, pp. 219-224, 1998.

[7] M. L. Wang, "Solitary wave solutions for variant Boussinesq equations," Physics Letters A, vol. 199, no. 3-4, pp. 169-172, 1995.

[8] M. Wang, Y. Zhou, and Z. Li, "Application of a homogeneous balance method to exact solutions of nonlinear equations in mathematical physics," Physics Letters A, vol. 216, no. 1-5, pp. 67-75, 1996.

[9] A.-M. Wazwaz, "The extended tanh method for new solitons solutions for many forms of the fifth-order KdV equations," Applied Mathematics and Computation, vol. 184, no. 2, pp. 10021014, 2007.

[10] S. Liu, Z. Fu, S. Liu, and Q. Zhao, "Jacobi elliptic function expansion method and periodic wave solutions of nonlinear wave equations," Physics Letters A, vol. 289, no. 1-2, pp. 69-74, 2001.

[11] J.-H. He and X.-H. Wu, "Exp-function method for nonlinear wave equations," Chaos, Solitons \& Fractals, vol. 30, no. 3, pp. 700-708, 2006.

[12] J. Liu and K. Yang, "The extended F-expansion method and exact solutions of nonlinear PDEs," Chaos, Solitons \& Fractals, vol. 22, no. 1, pp. 111-121, 2004.

[13] W.-X. Ma and J.-H. Lee, "A transformed rational function method and exact solutions to the $3+1$ dimensional Jimbo-Miwa equation," Chaos, Solitons \& Fractals, vol. 42, no. 3, pp. 13561363, 2009.

[14] W.-X. Ma, H. Wu, and J. He, "Partial differential equations possessing Frobenius integrable decompositions," Physics Letters A, vol. 364, no. 1, pp. 29-32, 2007.

[15] W.-X. Ma and Z. Zhu, "Solving the (3+1)-dimensional generalized KP and BKP equations by the multiple exp-function algorithm," Applied Mathematics and Computation, vol. 218, no. 24, pp. 11871-11879, 2012. 
[16] W. X. Ma, "Bilinear equations, Bell polynomials and linear superposition principle," Journal of Physics: Conference Series, vol. 411, Article ID 012021, 2013.

[17] W. X. Ma, “Generalized bilinear differential equations," Studies in Nonlinear Sciences, vol. 2, pp. 140-144, 2011.

[18] L. Wei, "A function transformation method and exact solutions to a generalized sinh-Gordon equation," Computers \& Mathematics with Applications, vol. 60, no. 11, pp. 3003-3011, 2010.

[19] L. Wei, "Exact solutions to a combined sinh-cosh-Gordon equation," Communications in Theoretical Physics, vol. 54, no. 4, pp. 599-602, 2010.

[20] M. V. Demina and N. A. Kudryashov, "From Laurent series to exact meromorphic solutions: the Kawahara equation," Physics Letters A, vol. 374, no. 39, pp. 4023-4029, 2010.

[21] M. V. Demina and N. A. Kudryashov, "On elliptic solutions of nonlinear ordinary differential equations," Applied Mathematics and Computation, vol. 217, no. 23, pp. 9849-9853, 2011.

[22] M. V. Demina and N. A. Kudryashov, "Explicit expressions for meromorphic solutions of autonomous nonlinear ordinary differential equations," Communications in Nonlinear Science and Numerical Simulation, vol. 16, no. 3, pp. 1127-1134, 2011.

[23] N. A. Kudryashov, D. I. Sinelshchikov, and M. V. Demina, "Exact solutions of the generalized Bretherton equation," Physics Letters A, vol. 375, no. 7, pp. 1074-1079, 2011.

[24] N. A. Kudryashov and D. I. Sinelshchikov, "Exact solutions of the Swift-Hohenberg equation with dispersion," Communications in Nonlinear Science and Numerical Simulation, vol. 17, no. 1, pp. 26-34, 2012.

[25] D. F. Lawden, Elliptic Functions and Applications, vol. 80 of Applied Mathematical Sciences, Springer, New York, NY, USA, 1989.

[26] Z. Yan, "An improved algebra method and its applications in nonlinear wave equations," Chaos, Solitons \& Fractals, vol. 21, no. 4, pp. 1013-1021, 2004.

[27] B. B. Kadomtsev and V. I. Petviashvili, "On the stability of solitary waves in weakyly dispersive media," Soviet Physics. Doklady, vol. 15, no. 16, pp. 539-541, 1970.

[28] Z. Qiao and J. Li, "Negative-order KdV equation with both solitons and kink wave solutions," EPL, vol. 94, no. 5, Article ID 50003, pp. 1-5, 2011.

[29] Y. Hase and J. Satsuma, "An N-soliton solution for the nonlinear Schrödinger equation coupled to the Boussinesq equation," Journal of the Physical Society of Japan, vol. 57, no. 3, pp. 679682, 1988.

[30] E. Fan and J. Zhang, "Applications of the Jacobi elliptic function method to special-type nonlinear equations," Physics Letters A, vol. 305, no. 6, pp. 383-392, 2002. 


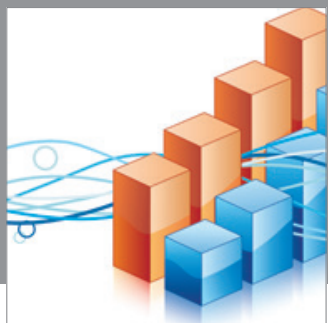

Advances in

Operations Research

mansans

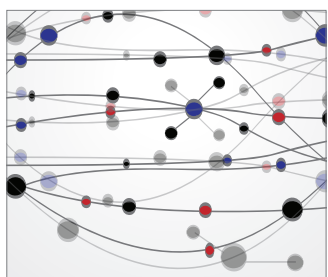

The Scientific World Journal
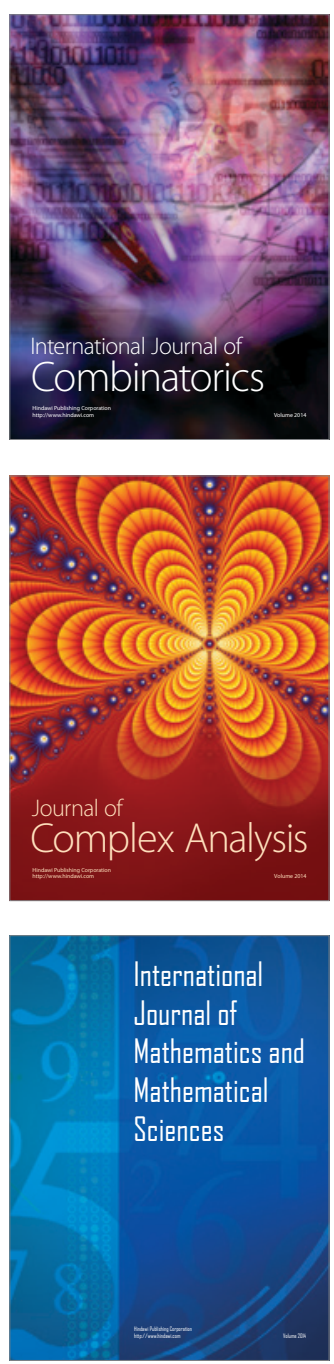
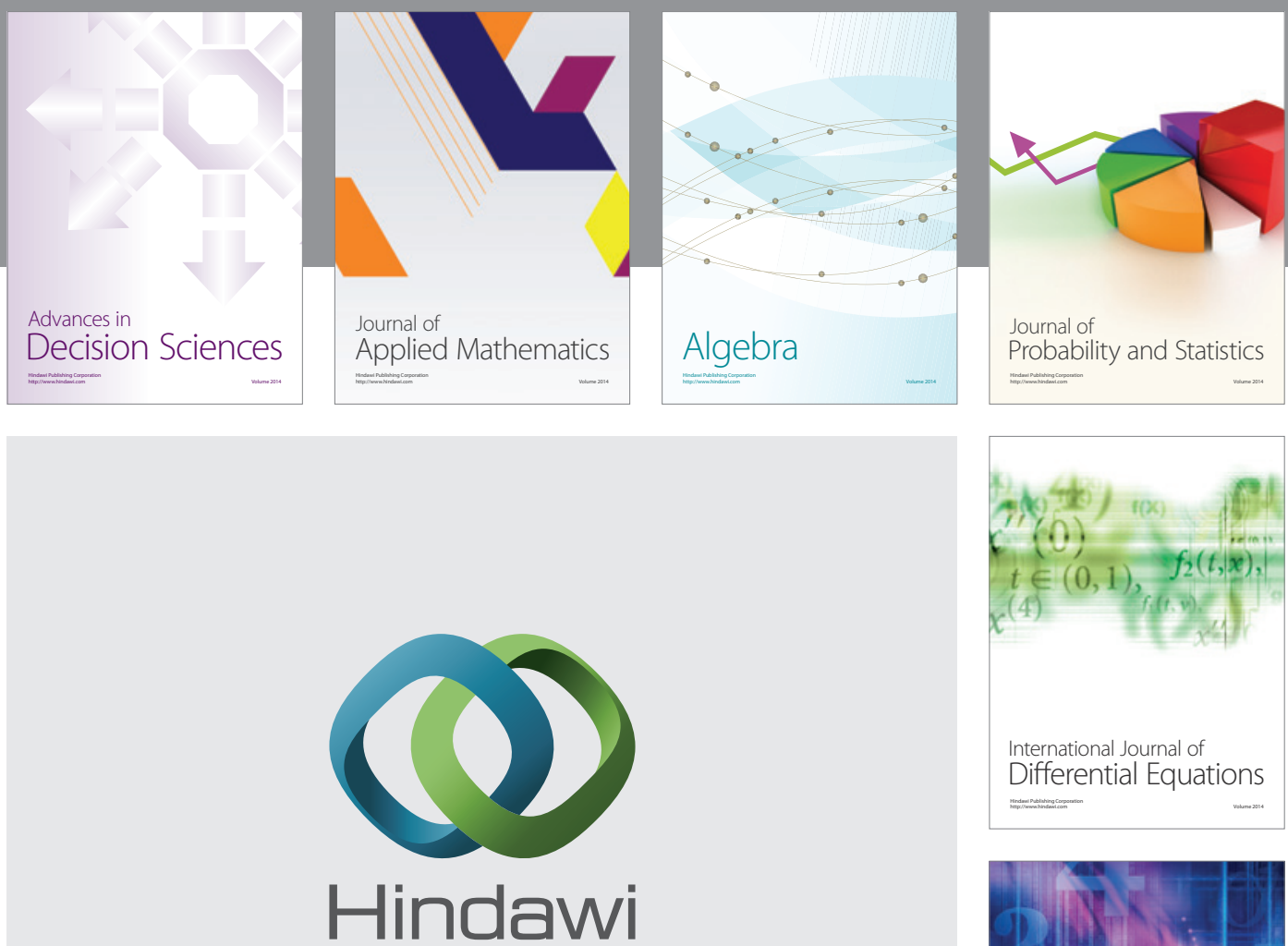

Submit your manuscripts at http://www.hindawi.com
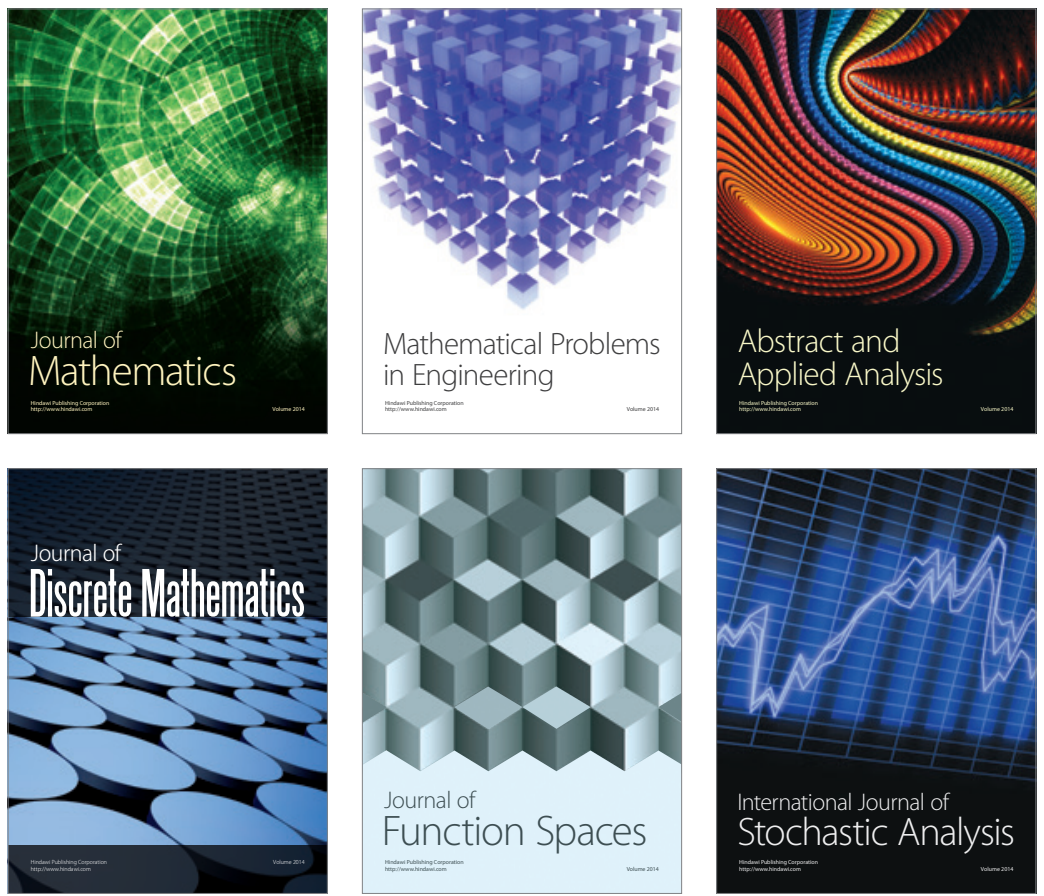

Journal of

Function Spaces

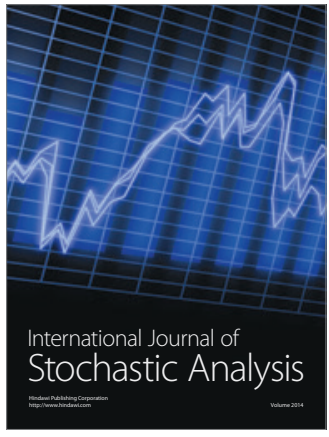

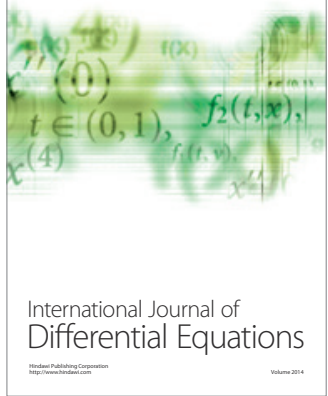
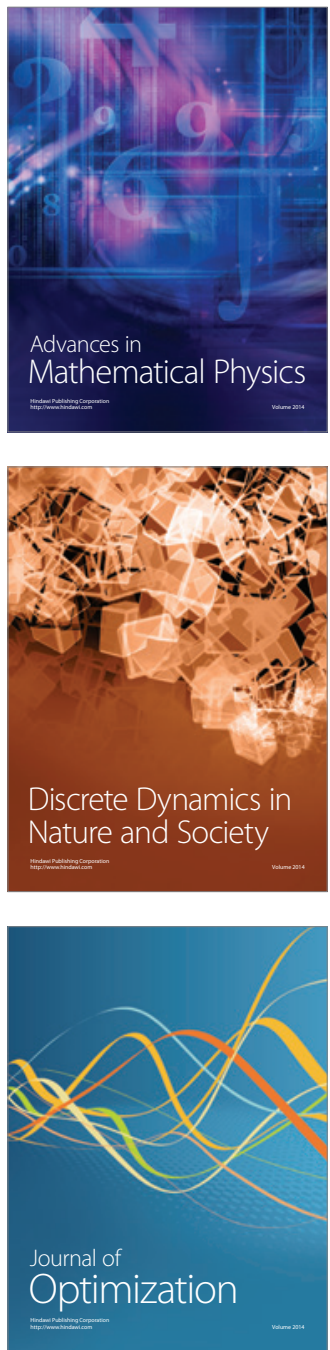